Heike Gfrereis*

\section{Das ist kein Papier}

\author{
Die Marbacher Literaturmuseen
}

DOI 10.1515/bfp-2017-0002

Zusammenfassung: Das Literaturmuseum der Moderne und das Schiller-Nationalmuseum zeigen die Bestände des Deutschen Literaturarchivs Marbach. Das heißt vor allem: viel Papier, viel Schrift und vieles, was nicht sichtbar ist den Kern des Schreibens und Denkens, die Energiezentren unseres Erfindungsdrangs. Die ausgestellte „Flachware“ ist so immer auch ein Drittes zwischen Körper und Geist, Material und Sinn, Zeichen und Sprache.

Schlüsselwörter: Marbach/Literaturarchiv; Literaturausstellung; Raumbild; Manuskripte; Schreibverfahren; Material

\section{This is not Paper. The Museums of Literature in Marbach}

Abstract: The Museum of Modern Literature and the Schiller National Museum exhibit the collections of the German Literature Archive. That means: much paper, much pieces of writing and many invisible things - the nucleus of writing and thinking, the motivations and dynamic environments of inventing. The "flat ware" in the exhibitions is a liminal area between body and mind, material and sense, sign and language.

Keywords: Marbach/literature archive; exhibition of literature; scenography; manuscripts; processes of writing; material

Die Ausstellungen, die das Deutsche Literaturarchiv Marbach seit 2006 in zwei Museen präsentiert - dem damals neu eröffneten Literaturmuseum der Moderne und dem 2009 wiedereröffneten sanierten Schiller-Nationalmuseum von 1903 -, haben alle Papier gezeigt: Manuskripte zumeist, Briefe, Zeichnungen am Rande, auch Bücher, vor allem gelesene oder in irgendeiner Weise beschriebene Bücher. Das Papier, selbst das scheinbar unbeschriebene, war immer ein Papier, auf dem jemand Spuren hinterlassen hatte: Zeichen, in den meisten Fällen Schrift, aber auch Fingerabdrücke, Tintenkleckse, Stempel, Flecken

*Kontaktperson: Prof. Dr. Heike Gfrereis, gfrereis@dla-marbach.de und Löcher, Ruß und Risse, Aufgeklebtes oder auch Eingedrücktes.

In allen Ausstellungen wurde dieses Papier so gezeigt, dass es dinglich wurde: zu einem Material, das unmittelbar da ist - vor jeder Deutung und unabhängig von all den Bedeutungen, die durch Berührung und Beschreibung entstanden sind. Wir haben in den Ausstellungen sehr oft das Zeigen vom Vermitteln getrennt und das Papier als Exponat inszeniert: in seiner ganzen Merk- und Fragwürdigkeit, auch seiner bescheidenen Alltäglichkeit und meist in seiner ganzen Erscheinung, von allen Seiten aus und mit all seinen Blättern. Die Raumbilder der Ausstellungen sind nahezu alle mit dem Material Papier entstanden und habe es jeweils auf sehr unterschiedliche Weise präsentiert, in ganz verschiedenen Anordnungen und Kombinationen. Auf einen an traditionellen Größen wie Autor, Epoche oder ,Gehalt" ausgerichteten semantischen, illustrierenden Überbau - ein Raumbild etwa, das das Manuskript von Berlin Alexanderplatz mit historischen Bildern vom Alexanderplatz hinterfängt, mit dem Film einer Straßenbahnfahrt durch Berlin, mit der Tonaufnahme einer Dampframme oder, der Collage zuliebe, lauter abgehängten Scheren haben wir verzichtet. Die Farben, Formen und Materialien dieser Ausstellungen waren höchstens semantisch in Bezug auf ihren Gegenstand und unsere Fragen an ihn. Sie waren Versuchsanordnungen, die dieses Papier ausnüchtern und zugleich auch etwas aus ihm herauslocken wollten, was wir nur mit ihm sehen und wissen können. Diese Reduktion war kein Kunstgriff, um die Aura der Exponate oder zumindest deren Kunstwert zu steigern. Wir wollen, dass die Besucher nur wissen müssen, aber auch wissen können, was sie selbst sehen können. Unsere Begeisterung für das, was uns dieses Papier alles zeigen kann, soll anstecken. Dieses so strenge wie emphatische „Das ist alles Papier“ ist immer auch ein „Das ist kein Papier“ - ein Spiel mit den Konventionen des Museums und der Kunst, die da, wo sie Erwartungen unterläuft, erreichen kann, das sowohl das, was man sieht, wie das, was man nicht sieht, auf ungeheuerliche Weise gegenwärtig ist. Ceci n'est pas une pipe. Doch was ist Papier, wenn es kein Papier ist? Einige der Ausstellungen aus den letzten zehn Jahren geben darauf beispielhafte Antworten ( 80 waren es insgesamt, kleinere und größere, gesehen von über 650000 Besuchern 
und vertieft in über 8400 Führungen und Literaturwerkstattprojekten).

\section{1 ... das Leben. Die erste Dauerausstellung im Literaturmuseum der Moderne}

In der ersten Dauerausstellung haben wir das Literaturarchiv sehr radikal ausgestellt, über 1300 Exponate, geordnet nach den Jahren ihrer Entstehung oder Benutzung und nach den Ablagekonventionen des Archivs in mehreren Lagen: Manuskripte, Bücher, Briefe und Karten sowie zugehörige Materialien, Bilder, Alben, Pässe, Schulhefte, Tagebücher und auch Nicht-Papiere - Erinnerungsstücke etwa und Schreibgeräte. Die Manuskripte lagen hier so nicht, wie oft in Literaturausstellungen, in einer vermeintlich ursächlichen, teleologischen oder zumindest logischen Konstellation, sondern in einer relativ natürlichen historischen Nachbarschaft. Sie waren gleichzeitig. Nicht mehr und nicht weniger. „In Marbach, und das macht die Ausstellung zum Ereignis, verschmelzen Ausdruck und Abdruck, versöhnen sich Leben und Text“", schrieb Ulrich Greiner in der Zeit. Für Uwe Wittstock war diese Ausstellung „ein Schaufenster ins Herz der Literatur“ (Die Welt) und für Julia Encke ein Eindringen in die „Dunkelkammern des Literarischen“ (Frankfurter Allgemeine Sonntagszeitung). Als das Literaturmuseum der Moderne 2007 mit dem höchsten britischen Architekturpreis, dem Stirling Prize, ausgezeichnet wurde, begründete die Jury das auch mit dieser Ausstellung: „Eine Treppe führt hinab in die Sammlungsräume mit ihrem notwendig dunkleren, künstlichen Licht. Es ist dieser Augenblick, in dem der Bau seine große Tradition zeigt - ein Gefühl des Eintretens in eine andere Welt, verbunden mit einer reichen, aber überlegten und konzentrierten Palette an Materialien und erfüllt von einem überwältigenden Oberlicht. Der Gang endet in der ständigen Ausstellung, wo sich eine magisch flimmernde Landschaft mit Manuskripten in gläsernen Vitrinen erstreckt. Der Raum ist beherrscht von einer gewissen Theatralik. In den Spiegelungen und Brechungen des Lichts und den unterschiedlichen Schatten, die von den kleinen intensiven Lichtpunkten erzeugt werden, verbirgt sich die Seele der deutschen Fantasie.“

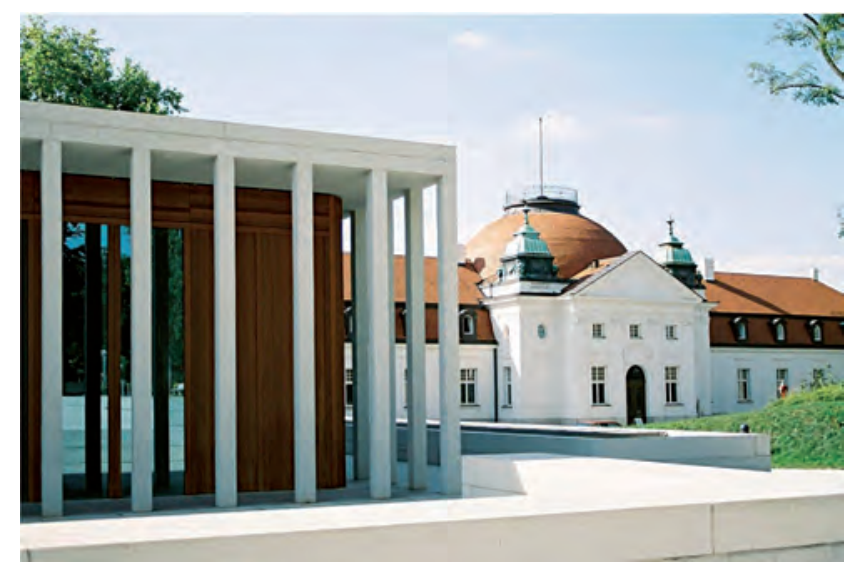

Abb. 1: Das Literaturmuseum der Moderne (LiMo) und das SchillerNationalmuseum (SNM) in Marbach

\section{2 ... Beruhigung und Versprechen: „Kippfiguren. Robert Gernhardts Brunnen-Hefte“}

675 Hefte, fast alle von der Firma Brunnen, hat Robert Gernhardt, der geheime Lieblingsdichter der Deutschen, vom Sommer 1978 bis zu seinem Tod im Juni 2006 gefüllt. Über 34000 Seiten mit Notizen in Wort und Bild, Gedichtentwürfen, Kunst-Theoretischem, Klatsch, Cartoons, Momentaufnahmen. Alle mit gelb-orangen BIC-Kugelschreibern festgehalten: die Gernhardt'schen Bären im Winterschlaf, die alte Hacke an der Mauer, das Hemd auf dem Stuhl auf der Terrasse kurz vor der Mittagszeit, der Mond in der Tür, die Eidechse auf der Fliese in der Sonne, die Katzen und Hund Bella, Gernhardts linke Hand mit Zigarette und ohne, die harten Striche und weichen Linien, die nicht schraffierten Beine und schattenlosen Figuren und jenes mir unvergessliche, lakonisch an nichts als eine Flasche Wein, einen Tisch und ein Männlein gebundene „Gut ist, wenn da Wein ist / Scheiße, wenn man klein ist“. Im Literaturmuseum der Moderne, eröffnet am 6. Juni 2006, an genau jenem Tag, an dem Robert Gernhardt in sein letztes Brunnen-Heft zu schreiben begann, war im Winter 2007/08 eine Auswahl der Hefte zu sehen. Eine weit gezogene Spirale, die sich durch die Ausstellungsräume zog: bunt auf der einen Seite, blau auf weiß auf der anderen. Nichts als Schulhefte, alle jedoch magische Instrumente, die eine besondere, eine distanziert-ästhetische, kreativironische, heitre Haltung gegenüber dem Leben mit sich bringen: Mit dem Heft auf den Knien und dem Kugelschreiber in der Hand lag Gernhardt unaufhörlich auf Lauer, um keinen Augenblick zu versäumen, der des Festhaltens wert ist. „Welch ein wunderbares Heft - / welch ein herrliches 


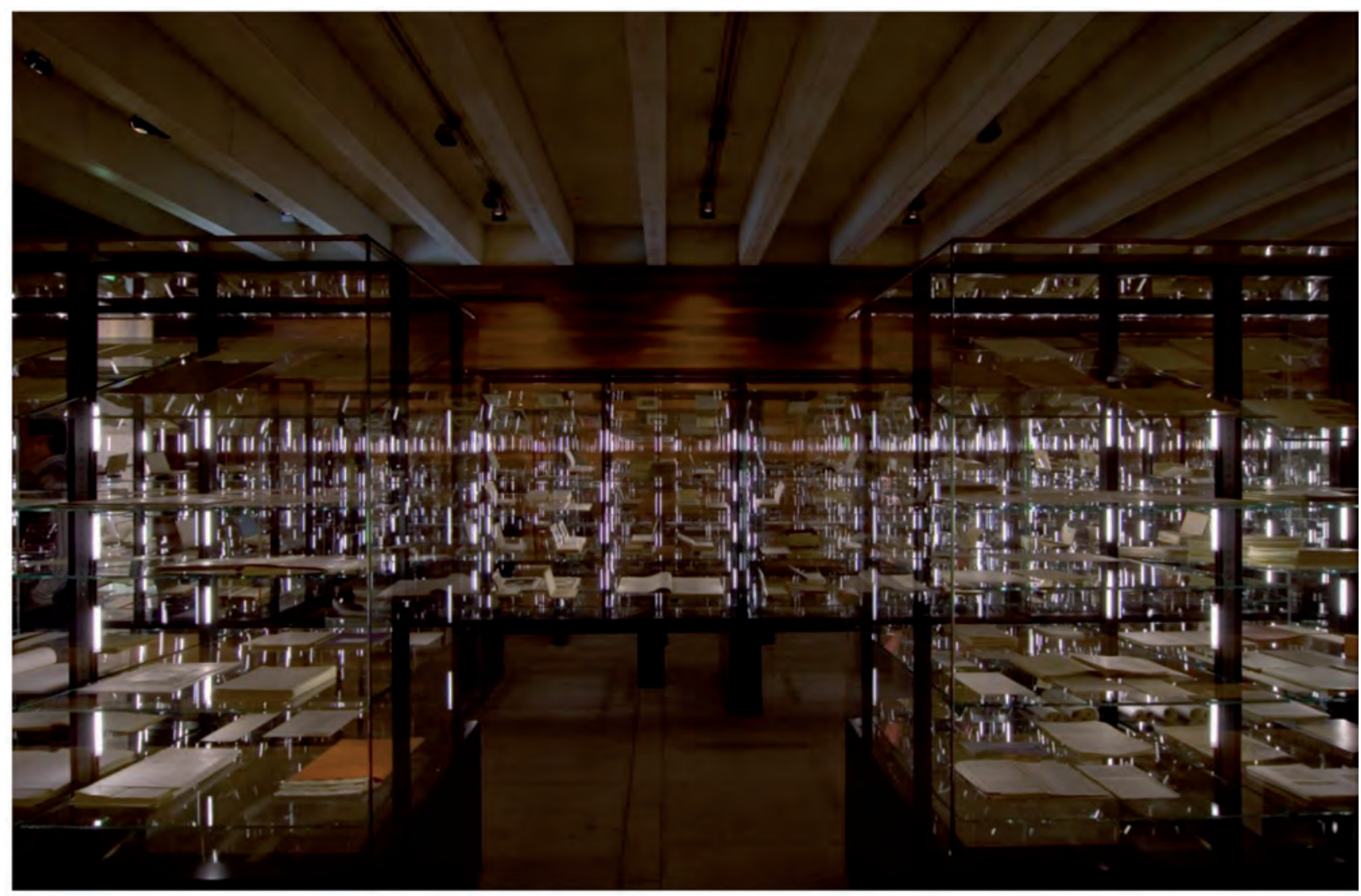

Abb. 2: Blick in nexus, die alte Dauerausstellung im Literaturmuseum Marbach

Geschäft - / dieses Heft hier vollzuschreiben / da zu sein und tollzubleiben / denkend; machend, / lenkend, lachend, / sinnend, kritzelnd / spinnend, witzelnd - / all das klingt so grauenhaft, / daß es die schönsten Frauen schafft. / Die klügsten Männer flohn bereits, / bei Zeile zwo in Gram und Schweiz“. Durch zwei Räume zogen diese Hefte ihre konstante Linie: Jedes eine Welt für sich und alle zusammen Beruhigung und Versprechen zugleich - für den Autor wie für seine Leser.

\section{3 ... Schweigen. Die Dauerausstellung im Schiller- Nationalmuseum}

Die historischen, 2009 mit einer neuen Dauerausstellung eröffneten Räume im Schiller-Nationalmuseum sind vollkommen anders gebaut als die Ausstellungsräume im Literaturmuseum der Moderne. Diese für das offizielle Exponieren von empfindlichen Archivalien, jene gehören zum idealen privaten Landhaus eines Dichterfürsten. Entsprechend verschieden in Inhalt und Gestaltung sind die
Dauerausstellungen - bei allen Gemeinsamkeiten: Beide sind Archivausstellungen und aus diesem Grund unvermeidbar dunkel und kühl; beide legen den Fokus auf das, was an diesen empfindlichen Archivalien sichtbar und zu entdecken ist, auf Mikroformen, auf Spuren literarischer Verfahren, auf Veränderungen von Zeichensystemen, Vorstellungs- und Ausdruckswelten, Sprech- und Darstellungshaltungen, auf Körperspuren und Zeitstempel. In beiden gibt es keinen vorgeschriebenen Rundgang, den ein Besucher ,abarbeiten' muss. Wer im Schiller-Saal steht, der hat die Wahl zwischen einem Schiller- und einem Thementeil. Die Räume im Schiller-Flügel setzen wie ein Kaleidoskop das Phänomen Schiller aus unterschiedlichen Bausteinen zusammen und laden zum hin und her gehen zwischen Körperbild und Körperspur, Schreiben und Leben ein. Die Räume im Themen-Flügel („Energie und Schrift“, „Ursprung“, „Liebe und Wahnsinn“, „Kleine Formen“) folgen einer heuristischen wie historischen Chronologie: Sie führen vom Kleinsten, Mikroskopischen - den Satz- und Schriftzeichen - zu poetischen Modellen der Weltentdeckung und -erklärung, von der Sprech- zur Lebenshaltung, aber auch vom Zeitalter der Empfindsamkeit zu Nietzsches Nihilismus, von der Französischen Revolution über 


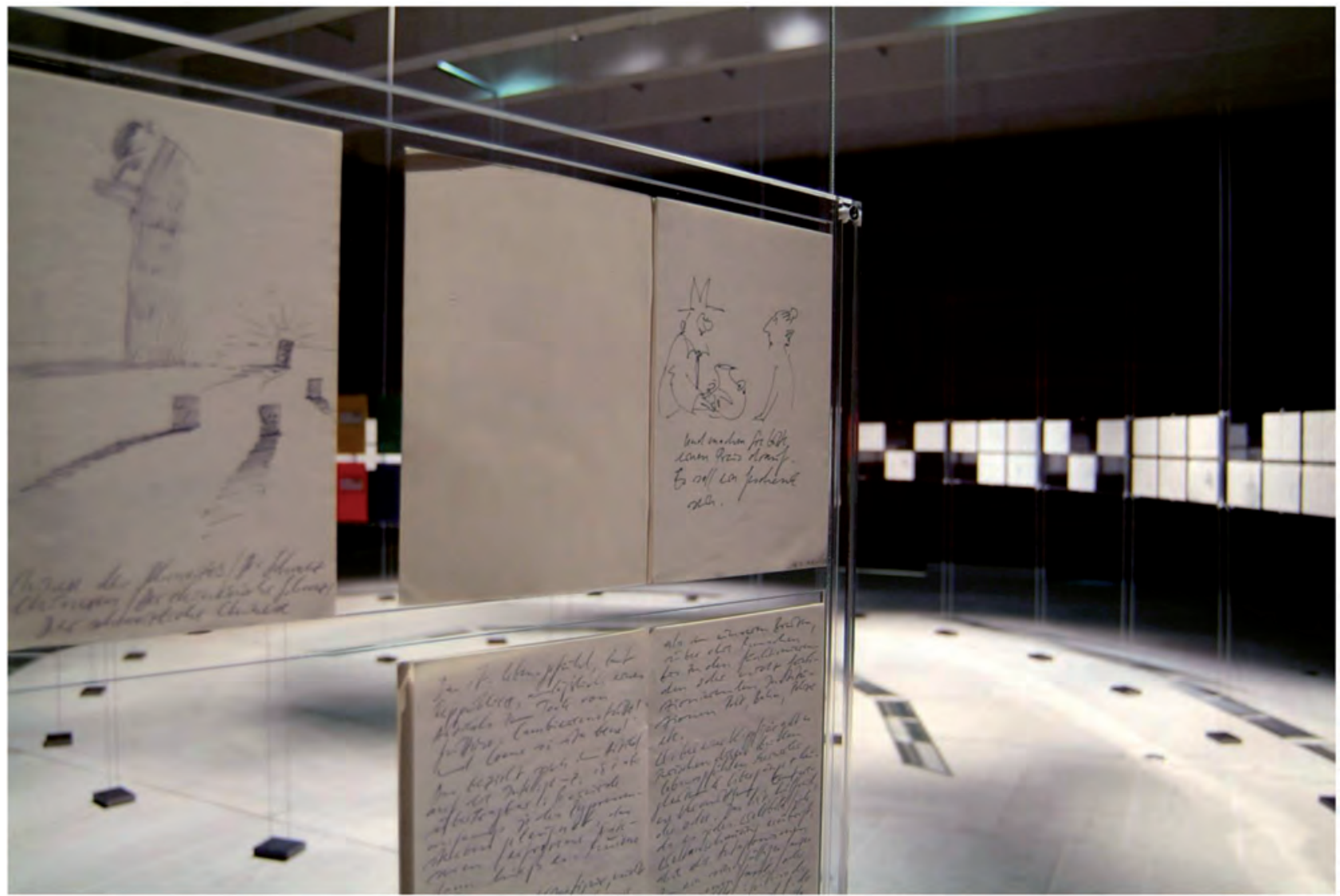

Abb. 3: Kippfiguren. Robert Gernhardts Brunnenhefte/2007 im Literaturmuseum der Moderne

die Napoleonischen Kriege, die Restauration und 48er-Revolution hin zur Gründung des Deutschen Reichs 1871. Jeder der neun Ausstellungsräume im Schiller-Nationalmuseum besitzt sein eigenes Raumbild und seinen besonderen Zugriff, seine auf den ersten Blick erfassbare besondere Perspektive auf die Dinge eines Archivs. Jeder zeigt eine Möglichkeit, die Archivalien mit ihrer Deutung und Vermittlung in ein Verhältnis zu setzten: figürlich, als Abdruck eines Körpers, chronologisch, als Überbleibsel eines Lebenslaufs, situativ, als Teil der Dichterwerkstatt, poetisch, als unmittelbare Türen zu einer nur imaginär vorhandenen Welt, oder historisch, als Quellen, die auch das, was uns bekannt und nah erscheint, im Horizont einer fremden Zeit verankern. Jeder gibt Antworten darauf, wie Schrift und Papier zu individuellen Ausdrucksmedien und zu Stellvertretern von Mimik und Gestik, von ganzen Menschen werden konnten und warum dadurch im 18. Jahrhundert die Literatur zur heiligen Poesie und die Autoren zu Dichterfürsten wurden und in der Konsequenz die Nachlässe von Dichtern in Archiven gesammelt und in Museen gezeigt werden. Dabei kehrt ein Motiv immer wieder, das für das 18. Jahrhundert von großer Bedeutung war: die Eroberung des Papiers als abgegrenzter, aber freier Platz für das
Denken, das Fühlen, das Sprechen und vor allem auch das Schweigen.

\section{4 ... Resonanz: „Wandernde Schatten. W. G. Sebalds Unterwelt“}

Literaturausstellungen können eine Textualität exponieren, die spezifisch räumlich ist und die in ihrer Dynamik, ihrer realen Präsenz und ihren ungeheuren Ausmaßen nur im Medium der Ausstellung rekonstruiert und damit auch dekonstruiert und vermittelt werden kann. Sie können das tun, indem sie den reproduzierbaren Text zum Exponat machen oder ihn mit den Kontexten seiner Konstruktion, seinen Entstehungsstufen zum Beispiel oder - wie wir es 2008 bei W. G. Sebald getan haben - dem Horizont seiner spezifischen Bibliothek, zusammenbringen. Alles in Sebalds Nachlass ist mehrfach determiniert, alles steht in einer Relation zueinander und oft in mehr als nur einer, so dass sich zeitlich unterschiedliche Texte, Spuren und Bilder in einem ,tief' scheinenden Raum überlagern. Fakten 


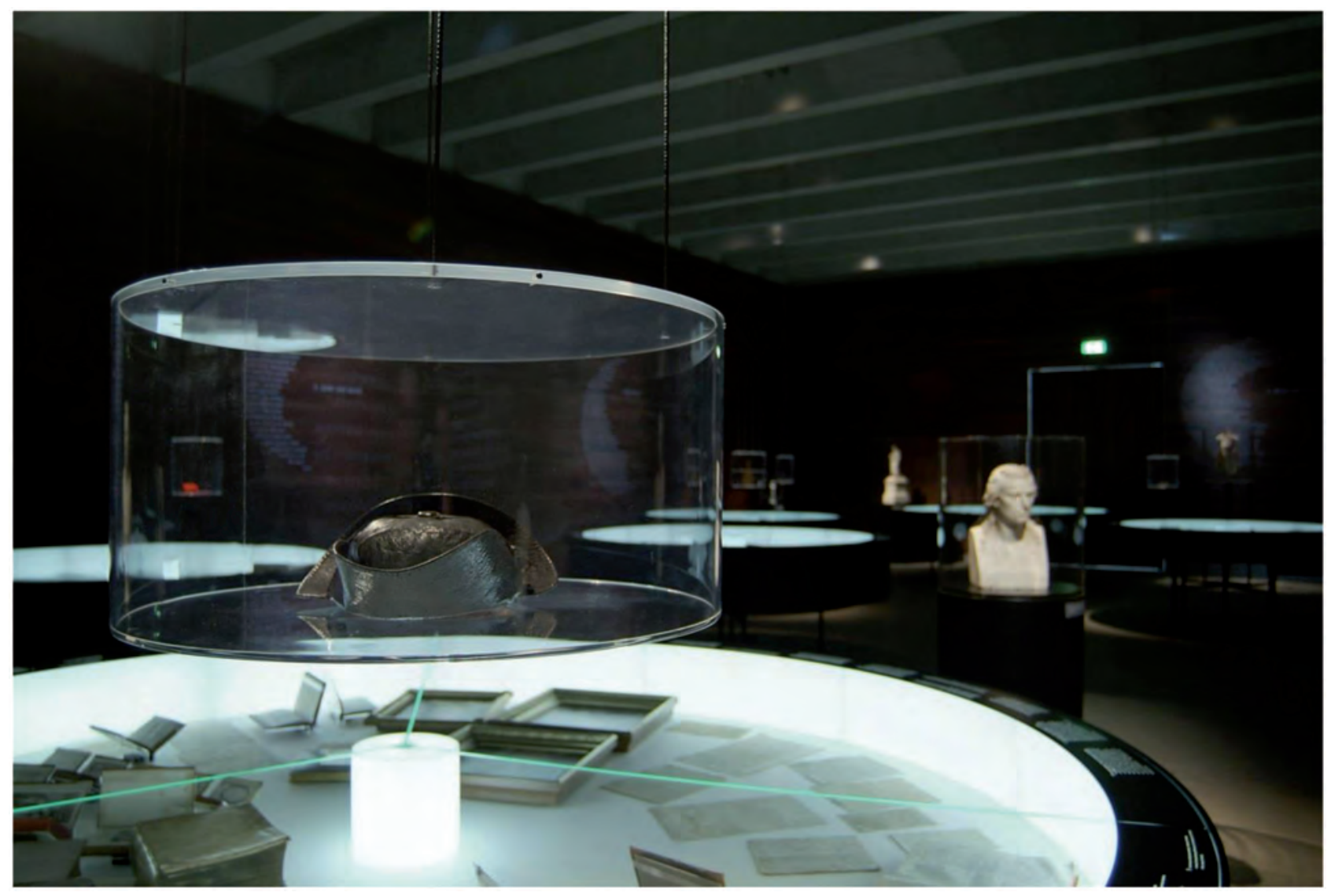

Abb. 4: Autopsie Schiller. Eine literarische Untersuchung (2009 im Literaturmuseum der Moderne)

sind hier Fiktionen und Fiktionen Fakten. Alles ist Literatur, wird Literatur, war Literatur; alles gehört in den Bereich der Textsubjekte, die ihr Leben in und mit dem Text entwickeln. Nichts, wenn man es so zuspitzen will, verrät den Autor als Menschen: Sebald zitiert Kafka, der wiederum Goethe zitiert, oder Robert Walser, der wiederum über Heinrich von Kleist schreibt. Nahezu alle ,authentischen', in seinen Texten abgebildete Objekte - wie zum Beispiel die Visitenkarte von Ambos Adelwarth - sind durch Bricolage entstanden und oft fingiert. Sebalds Bibliothek liefert für seine eigene Technik, Text durch Fotografien zu beglaubigen, eine einschlägige Inspirationsquelle: Er besaß zahlreiche rororo-Monographien. Die Ausstellung hat diese Maschinerie gleichsam in situ nachgebaut: Passagen aus vier seiner großen Erzählungen Schwindel.Gefühle, Die Ausgewanderten, Austerlitz und Die Ringe des Saturn wurden in mehreren Schichten untereinander mit den dazu in einer Beziehung stehenden Text- und Bildmaterialien kombiniert, so dass sich parallel zu den Haupttexten deren Subtexte und Tiefenstrukturen entfalten konnten, auch über die jeweiligen Erzählungen hinaus. Die Ausstellung hat sich an und aus dem Nachlassmaterial herausentwickeln können, das seine Zusammenhänge nahezu von alleine in den Vordergrund spielte, wenn man es nebeneinander legte: Sah man nur auf die zahlreichen Bilder, entdeckte man plötzlich, wie sich nahezu inflationär die Hände ausbreiteten, Augenpaare erschienen, Unschärfestellen, Gitterlinien, Schmetterlinge und Falter, Blumen und Pflanzen, Feuer und Eis. In der Spiegelreflexkamera von Jacques Austerlitz kehrt dann - vom Nachlasszusammenhang aus betrachtet - nicht nur die Bahnhofshalle von Antwerpen wieder, die Augenpaare der Raubvögel und von Améry und Wittgenstein, sondern auch das Augenleiden des Ich-Erzählers, die Augen auf dem Rad eines Pfaus, die beiden Kugeln auf einem Billardtisch und eine Versuchsanordnung von Goethe, mit der er herauszufinden versuchte, warum wir Farben sehen, die es nicht gibt („entoptische Farben"). Sichtbar verständlich wurde dieses Schreibverfahren, das nach Ähnlichkeiten und Ansteckungen sucht und durchaus auch ein wenig vampiristisch ist und das Leben raubt, um es der Literatur zu schenken. In den Psychopathographien des Alltags (1972) von Alexander Mitscherlich markiert Sebald: „er weiß zum Beispiel, daß er alles, was er seinen Büchern gibt, seinem Leben entzieht“. In Eine Insel in der Nähe von Magora (1973) von Lars Gustafssons: „Weil sich aber überhaupt nichts aussagen läßt, son- 
dern immer nur etwas anderes, das an die Stelle dessen tritt, was man sagen will, hat man am Ende die größte Lust, sich selber unsichtbar zu machen, damit wie durch einen Zauber die Welt in ihrer erschreckenden Einfachheit hervortrete“. Sebald selbst notiert sich (auf einem Blatt, das sich in seinem Nachlass erhalten hat): „Phantasie kommt nicht daher, daß man ein interessantes Leben führt.“

\section{Gegen-Zeit: „Ernst Jünger. Arbeiter am Abgrund"}

Die 2010 gezeigte Ausstellung zu Ernst Jüngers Arbeit am Text hat sich auf zwei Zeigetechniken konzentriert und diese auch als heuristische Methoden genutzt: serielle Reihung und metonymisch-metaphorische Kombination. Ein großes Vitrinenband exponierte den immensen, nahezu 90 Jahre umfassenden Zeitraum von Jüngers Schreibarbeit und legte seine Verfahren der Literarisierung, Systematisierung, Umcodierung, Neufassung und buchstäblichen Umformatierung seiner Tagebücher frei: Den auf den oben Vitrinenböden ausgestellten kleinen, meist in Heften und Büchern im Format DIN A5 oder auch A6 entstandenen Tagebüchern standen auf den unteren Böden die daraus hervorgegangenen, nahezu alle auf DIN A4-Blätter geschriebenen Werke gegenüber. Insgesamt rund 300 Tagebücher, Manuskripte, Typoskripte und ,Typogramme‘, der ganze erhaltene literarische Nachlass, vom ersten Tagebuch des Schülers bis zum letzten Notizkalender, vom Gedicht in der Schülerzeitung zu Siebzig verweht. Augenblick und Ausbreitung, Ereignis und Niederschlag, Einfall und Weiterverarbeitung, Initial und Übersetzung - die für Jünger konstante Schwelle zwischen Tagebuch und Werk durchschnitt als Horizont die 19 zwei Meter hohen, insgesamt 16,5 Meter langen Vitrinen. Die Übertragung von einem Tagebuch ins Manuskript des Werks folgt dabei gerade keiner einfachen chronologisch-teleologischen Linie. Wörter, Sätze und Bilder breiten sich aus, verschieben sich, wandern durchaus auch aus dem Werk zurück in die Tagebücher, münden in kein endgültiges Ziel. Jüngers Texte sind keine festen Entitäten mit Anfang, Mitte und Ende, sein Werk ist in Bewegung. Schreiben heißt bei ihm nicht Abschließen, sondern Öffnen, wiederholtes Neu-Schreiben, anders Fassen, Verflüssigen, momentanes Kristallisieren wie in einem Kaleidoskop. Die Besucher konnten entlang der Grenzlinie zwischen Tagebüchern und Manuskripten diese Arbeitstechnik überblicken und verfolgen, wie sich Daten, Wörter und Bilder aus den Tagebüchern nach unten in die größerformatigen Werke verschieben und verzweigen und $\mathrm{zu}$ neuen Zusammenhängen arrangierten. Jünger dreht zum Teil seine Tagebücher um und beginnt von hinten, oder er lässt sie nur halb gefüllt liegen und führt sie erst nach Jahren wieder fort. In ein Notizbuch mit Kalender von 1936, in dem er Nachträge aus dem Sommer 1940 abgearbeitet hat, schreibt er oben links das Programm: „Nota: Das Tagebuch ist noch zu überholen, unter anderem zu metallisieren!“ Am 3. Januar 1943 (drei Tage, nachdem er am letzten Tag des Jahres das Büchlein umgedreht und von der anderen Seite begonnen hat) bestimmt er dieselbe Technik mit einer Metapher, die Kälte und Härte durch Hitze verflüssigt: „Tagebuch: kurze kleine Notizen wie Tee in Krümeln; ich gieße später bei der Abschrift das heiße Wasser auf, das ihnen das Aroma erschlieBen soll.“

Vom ersten bis zum letzten Tagebuch breitet sich bei Jünger eine Welt immer wiederkehrender einfacher Symbole aus: Augen, Sterne, Linien, Schlangen sowie die Unendlichkeits- und Unbestimmtheitszeichen $\infty$ und $\mathrm{x}$. Hinzu kommen in immer größer werdender Dichte Einklebungen: Blumen und Blätter, aber auch Eintrittskarten, Fotos, Zeitungsausschnitte, Postkarten, Schmetterlinge, Larven und sogar kleine Schlangen. Jünger hält immer wieder Ähnliches fest, den Ort und den Tag, an dem er zu schreiben anfängt und aufhört, den Ort und Tag, an dem er die Einklebungen fand - Zahlen und Plätze, Ziffern und Namen werden durch ihre Ritualisierung austauschbar. Er setzt das Schreiben gegen die Zeit und legt mit Papier ein Netz an Beziehungen aus, das weit über die einzelnen Texte hinausgeht - eine Art idealer Raum des Assoziierens, eine Werkstatt der Arbeit an einem eigenen mythischen System.

\section{Idee und Tat: „Autopsie Schiller. Eine literarische Untersuchung“"}

Auffällig viele Spiele sind von Schiller überliefert. Kartenspiele, Legespiele, Würfelspiele und ein Schachspiel. An Ludwig Ferdinand Huber schreibt er am 5. Oktober 1785: „Gewöhnlich machen wir den Fehler, die Zukunft nach einem augenbliklichen höhern Kraftgefühl zu berechnen, und den Dingen um uns her die Farbe unsrer Schäferstunde zu geben. Ich lobe die Begeisterung, und liebe die schöne ätherische Kraft, sich in eine große Entschliessung entzünden zu können. Sie gehört zu dem beßern Mann, aber sie vollendet ihn nicht. Enthousiasmus ist der kühne kräftige Stoß, der die Kugel in die Luft wirft, aber derjenige hieße ja ein Thor, der von dieser Kugel erwarten wollte, daß sie ewig in dieser Richtung und ewig mit dieser Geschwindigkeit, auslaufen sollte. Die Kugel macht einen Bogen, denn ihre Gewalt bricht sich in der Luft. Aber im süßen Moment der 


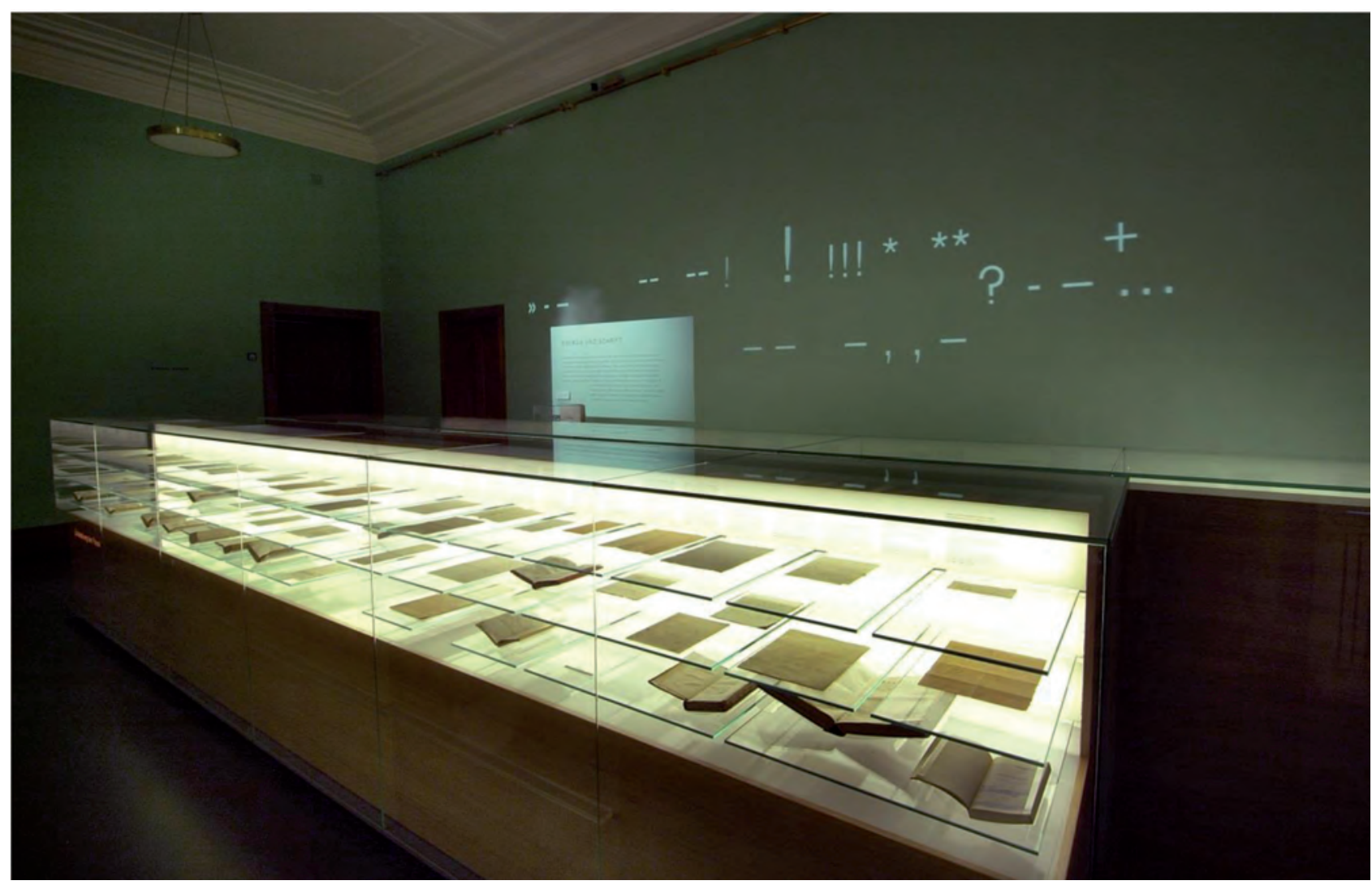

Abb. 5: Kapitel „Schrift und Energie“ in der Dauerausstellung im Schiller-Nationalmuseum

idealischen Entbindung pflegen wir nur die treibende Macht, nicht die Fallkraft, und nicht die widerstehende Materie in Rechnung zu bringen. [...] Wenigstens wollen wir Arm in Arm biß vor die Fallthüre der Sterblichkeit dringen, wo die Linien zwischen Menschen und Geistern gezogen sind. Enthousiasmus bleibe stets unsre erste treibende Gewalt, unsre Kugel soll wenigstens so kräftig von der Hand empor fliegen, daß der Bogen in den Wolken verschwinden, und ihr Rükfall kaum mehr geglaubt werden soll." Der Vorteil der Kunst des Schreibens, die bei Schiller häufig mit einer buchstäblichen Linie auf dem Papier verbunden ist, liegt darin, dass man diese Bahn selbst und an jedem Punkt steuern kann.

In der 2009 gezeigten Ausstellung wurden Dinge ganz unterschiedlicher Art - Manuskripte, Briefe, Bücher, Erinnerungs- und Kleidungsstücke von Friedrich Schiller - so in runden Vitrinen gelegt, als würde man mit Karten spielen, Patiencen legen oder Bilderrätsel bauen: nebeneinander, oft in scheinbar unvermittelter, assoziativer Konfrontation. Die Raumfolge orientierte sich an der Topografie des menschlichen Körpers, nicht an den Zeitpunkten und Orten der Biografie. Sie nahm buchstäblich den Weg vom Kopf bis zum Fuß des Dichters. Nur selten markierten die Nachbarschaften biografische oder werkgenetische Zusammenhän- ge, vielmehr steckten sie historische Wort- und Bildfelder ab, poetische und alltägliche, sinnliche und intellektuelle, seelische und körperliche Bedeutungsmöglichkeiten, Erinnerungs- und Imaginationsräume um 1800. Das Papier war das wichtigste Element dieses Tauschverkehrs, der Denken und Schreiben, Idee und Tat verknüpfte. Dem Freund Christian Gottfried Körner macht Schiller zum Beispiel am 10. bzw. 22. Februar 1785 eine schicksalhafte zwölftägige Unterbrechung mit zahlreichen, um genau zu sein: mit zwölf Strichen auf dem Briefpapier deutlich: „(Hier bin ich neulich durch einen unvermuteten Besuch unterbrochen worden, und diese 12 Tage ist eine Revolution mit mir und in mir vorgegangen, die dem gegenwärtigen Briefe mehr Wichtigkeit gibt, als ich mir habe träumen laßen - die Epoche in meinem Leben macht)“.

\section{7 ... Berührung: „Franz Kafka. Der ganze Prozess“}

Der Abdruck, der Stempel und die Spur - die Signale einer konkreten, authentifizierenden Berührung - sind Elemente dieses Zeitraums, den das Original eröffnet. Sie finden 


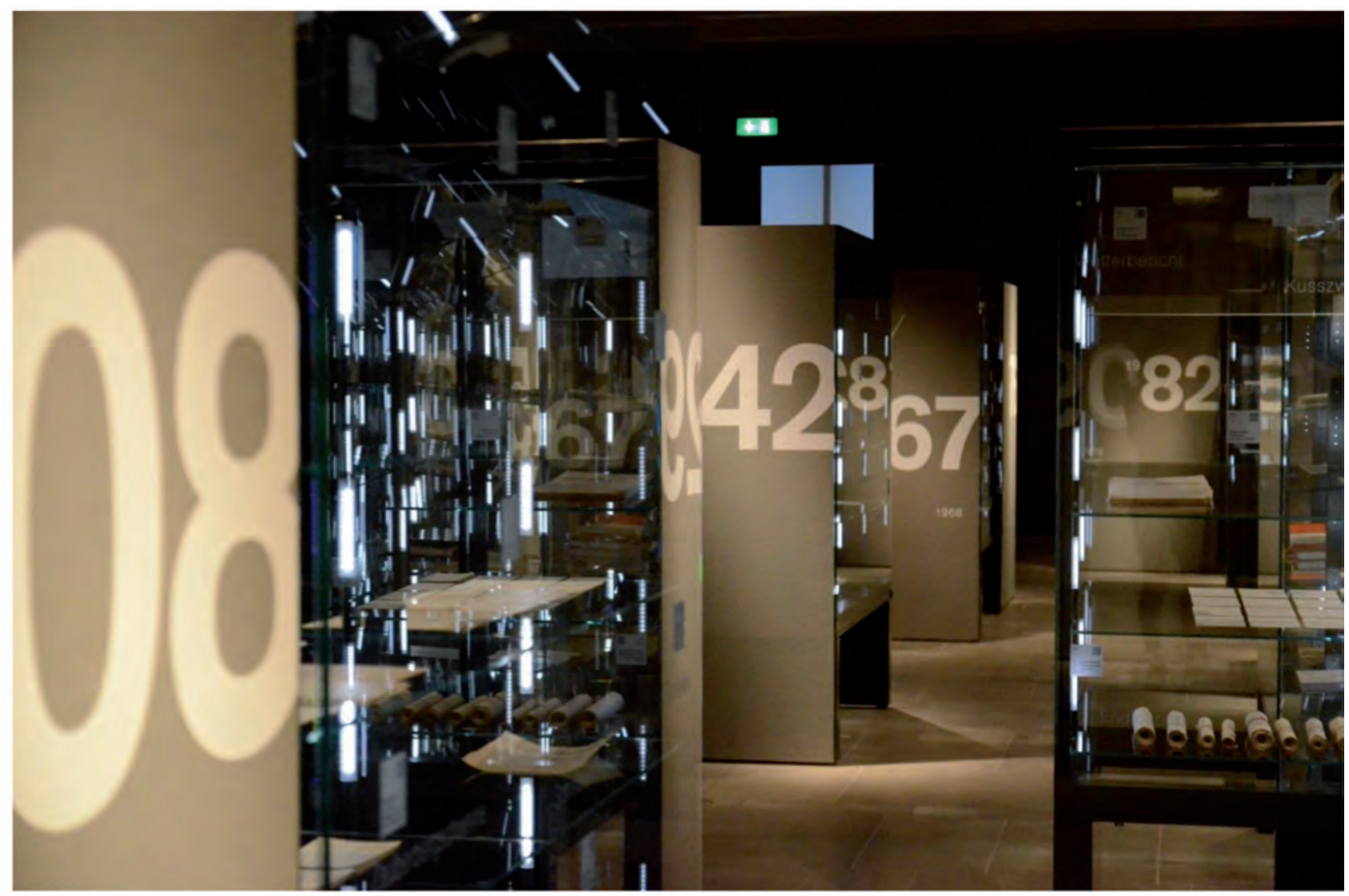

Abb. 6: Blick in Die Seele, die neue Dauerausstellung im Literaturmuseum der Moderne

sich natürlich auch in Kafkas Manuskript zu seinem Roman Der Prozess, in dem die Spuren des Autors, des Herausgebers und des Setzers zu finden sind. Am prominentesten sicher die erste Streichung auf der ersten Manuskriptseite und die letzte Streichung auf der letzte. Beide Streichungen zeigen, wie Kafka die jeweiligen semantischen Felder auflädt und enger zieht, indem die Formulierung „wurde verhaftet“ das unpräzise „war gefangen“ ersetzt und „[d]ie Scham sollte ihn überleben“ an die Stelle von „Scham war sein letztes Lebensgefühl“ rückt. Der Prozess wird Josef K. auf diese Weise von Anfang bis zum Ende gemacht. Im Idealfall lesen wir im und durch das Original genauer, sorgfältiger, Wort für Wort und Satzzeichen für Satzzeichen, weil wir es so lesen können, wie es geschrieben worden ist. Bei Kafka heißt das, sich keiner Schreibweise und Lesart sicher sein zu können, weil er selbst einen einzelnen Buchstaben wie das „K.“ beständig variiert und auch $\mathrm{zu}$ „F.“ verschreibt. Unsere kulturell konditionierte Erwartung an Literatur, dass sie eine Bedeutung oder eine übersetzbare (Selbst-)Referenzialität hat, wird hier enttäuscht, aber auch genährt, indem im Prozess dem Text selbst der Prozess gemacht wird. Schon auf der elften Manuskriptseite des von Max Brod als erstes Kapitel interpretierten Konvoluts ist die Anschuldi- gung zu lesen: „Sie wollen einen Sinn und führen das Sinnloseste auf was es gibt! Ist es nicht zum Steinerweichen?" Das Manuskript verhält sich mimetisch zum Roman, der sich immer wieder auf die Arbeit am Text und das Schreiben bezieht. Wir sehen am Original, warum geschrieben worden ist, was wir lesen. Wir schreiben quasi den Text noch einmal und vollziehen alle Schreibprozesse und -gesten nach: Wortauswahlvorgänge, Ausschweifungen, Abbrüche, Vor- und Umordnungen, die ganze Mimikry des Schreibers, sein Tempo und seine Pausen, den Atem des Schreibens. Kafka hat den Prozess wie die meisten seiner Texte nicht von vorne nach hinten geschrieben, sondern in einer großflächigen Parallelaktion. In insgesamt zehn Schreibhefte mit meist 40 Blättern notiert er die Teile seines Romans: durcheinander, das Ende nah beim Anfang, den Anfang wiederum verteilt auf drei Hefte. Durchmischt mit Tagebucheinträgen und immer wieder neu begonnen oder abgebrochen finden sich hier die Erzählungen Die Pferde von Elberfeld, Blumfeld, ein älterer Junggeselle, Der Dorfschullehrer und Der Hungerkünstler, Erinnerungen an die Kaldabahn, Das Schloss und Der Verschollene sowie die berühmten Tagebucheinträge aus dem Juli und August 1914. Sie alle stehen vor, zwischen oder nach den Prozess-Blättern. Kafka löst später die Prozess- 
Passagen aus den Heften und bündelt sie zu 16 Konvoluten. Max Brod stellt dann nach dessen Tod die erste Leserordnung her, indem er die Blätter im Manuskript in eine Reihenfolge bringt, die sie heute noch haben. So besitzen die Manuskript-Blätter jeweils ganz unterschiedliche Blattnachbarn: a) die ursprünglich in einem Heft direkt anschließenden Blätter, sozusagen die Nachbarn des Schreibens, b) die Anschlussblätter in Kafkas Konvoluten, also die Nachbarn, die Kafka dann zueinander gebracht hat, und c) diejenigen, die für Brod am ehesten einer Satz- und Textlogik entsprachen, die Roman-Nachbarn. Originale sind auch erratische Blöcke, Widerstände: unlogisch, intransparent und antimimetisch. Im besonderen Fall von Kafka geht das mit der ,kafkaesken Logik' seiner Erzählung zusammen und kann dann also, zur Freude vieler Interpreten, als Autoreferenzialität doch wieder mimetisch interpretiert werden. In der Marbacher Prozess-Ausstellung 2013 hat man keinen wie Perlen aufgereihten Papierschatz gesehen, kein Gut von national-politischer Bedeutung. Man konnte sehen, was da ist: Die Karten waren auf den Tisch gelegt, 161 Blätter mit 14 Deckblättern. Man konnte sehen, an wie vielen imaginären Schreibtischen Kafka gleichzeitig gearbeitet hat, sah seine Textlager und Papierberge und die eigenwillige Art, in der er jedes selbst gewählte Schema, allen voran das immer gleiche Manuskriptformat, immer wieder unterlaufen hat. Um das zu sehen, musste man als Besucher nicht mehr lesen als den kleinen Einführungstext. Was ein bedeutungs- und auch strukturoffener Text ist, ein Text ohne Anfang, Mitte und Ende, ein moderner Text, das ist in dieser Ausstellung sichtbar verständlich geworden. Man konnte etwas sehen, was man auf diese Weise nur in dieser, auf eine kurze Zeit bemessenen Ausstellung erfahren konnte, an diesem Ort und in dieser Atmosphäre, mit diesen durch das Ausstellungslicht und das Vitrinenglas und das Exponat selbst erzeugten Schattierungen, Einfärbungen und Unschärfen.

\section{8 ... Seele. Die neue Dauerausstellung im Literaturmuseum der Moderne}

Papier ist auch vieles andere: Rohes (so zum Beispiel in der Ausstellung „Zettelkästen. Maschinen der Phantasie“), Anfang und Ende und Grund oder besessen (in „Der Wert des Originals“), Vorstellungs- und Strukturierungshilfe (in „Ordnung. Eine unendliche Geschichte"), Anheizer und Abkühler (in „Ich liebe Dich!“), Belichtungsmaterial und ästhetischer Rahmen (in „Fotos. Reisen von unterwegs“), Raum und Form und deren Auflösung und Negation (in
„Das bewegte Buch“). Von weißer Magie spricht Lothar Müller in seinem Buch über Papier. Bei der Neugestaltung der Dauerausstellung im Literaturmuseum der Moderne 2015 haben wir Dinge ausgesucht, die uns aufstören. Kleine, aber epochale Erscheinungen einer Literaturgeschichte im Archiv: Augenblicke, Einschnitte, Brenn- und Störpunkte. Auslöser von Zeit- und Raumerfahrungen, die mit dem Schreiben und Lesen einhergehen: Plötzlichkeit, Verdichtung, Dehnung, Dauer im Wechsel, Langeweile und Langsamkeit, Zähigkeit, Ziellosigkeit, Fremdheit und Unverständlichkeit, Versprechen, Vertröstung, Ablenkung, Erund Überfüllung, Unendlichkeit und Überschaubarkeit, Erleuchtung, Erlösung, auch Auslöschung. Manche Dinge aus dem Archiv tauchen immer wieder auf, spuken wie Geister. Intelligenzverstärker, Gefühlstreiber, Gedankenschärfer, Bildzünder. In anderen scheint uns der Geist der Zeit oder auch eine andere Seele zu begegnen: Gespenster derer, die sie besessen und meist auch gemacht haben von Hand, mit dem Stift, der Schreibmaschine, dem Computer. Klacken, Kratzen, Reiben, Rascheln, Flattern, Murmeln, vielleicht auch Klopfen und Schreien, das Befeuchten der Finger und Lippen, Luftholen, Ein- und Ausatmen sind die Klänge ihrer Entstehung. Die Zeit in ihrer so unterschiedlich empfundenen Dauer von Minuten, Stunden, Tagen, Wochen, die volle und die leere Zeit, die hier in die bloße Chronometrie einer Ordnung nach Jahren gefasst ist - sie ist der ständige Begleiter dieses Schreibens, das dank unserer Fantasie keine Bindung an Orte kennt, sehr wohl aber Widerstände, Störungen, Gegenseiten: Papiermangel, Schreibkrisen, Kriege, Vertreibung, Zensur, Krankheit, Liebe, Tod, Glück und Unglück. Dennoch. Trotzdem. Gerade weil. „Immer schreibt schon einer“, heißt es in einem von Robert Gernhardts Gedichten, und: „Immer schreibt noch einer“. 280 Exponate, nahezu alle aus Papier, zeigen sich hier in diesem Sinne.

Um diese Seele des Papiers sichtbar zu machen, ist die neue Dauerausstellung mehrschichtig aufgebaut. Kurzwörter auf dem Vitrinenglas geben uns eine Perspektive eine Stimme von vielen, poetisch und neugierig: Liebeslückentext, Regenwurmtechnik, Zauberberg, Schonschlafendschreiben.

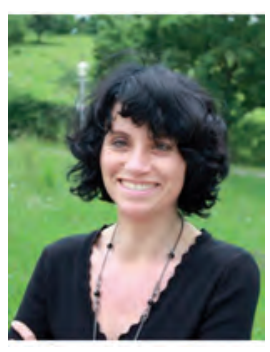

Prof. Dr. Heike Gfrereis

Deutsches Literaturarchiv Marbach

Leiterin Literaturmuseum der Moderne und Schiller-Nationalmuseum Deutsches Literaturarchiv Marbach Postfach 1162 D-71672 Marbach am Neckar gfrereis@dla-marbach.de 